\title{
The inverse scattering problem of some Schrödinger type equation with turning point
}

\section{Zaki FA El-Raheem* and Farouk A Salama}

${ }^{*}$ Correspondence: zaki55@Alex-Sci.edu.eg Department of Mathematics, Faculty of Education, Alexandria University, Alexandria, Egypt

\begin{abstract}
In this paper the inverse scattering problem is considered for a version of the one-dimensional Schrödinger equation with turning point on the half-line $(0, \infty)$. The scattering data of the problem is defined and the fundamental equation is derived. With the help of the derived fundamental equation, in terms of the scattering data, the potential is recovered uniquely.
\end{abstract}

MSC: 58C40; 34L25; 34B05; 47A40

Keywords: Schrödinger equation; scattering function; asymptotic formula; contour integration; main integral equation; uniqueness theorem; inverse scattering problem

\section{Introduction and preliminaries}

\subsection{Introduction}

Inverse problems of spectral analysis consist in recovering operators from their spectral characteristics. Such problems often appear in mathematics, mechanics, physics, electronics, geophysics, meteorology, and other branches of the natural sciences. Inverse problems also play an important role in solving nonlinear evolution equations in mathematical physics. Interest in this subject has been increasing permanently because of the appearance of new important applications, and nowadays the inverse problem theory is developed intensively all over the world. The greatest success in spectral theory in general, and in particular in inverse spectral problems, has been achieved for the Sturm-Liouville operator $y:=-y^{\prime \prime}+q(x) y$, which also is called the one-dimensional Schrödinger operator.

The main results on inverse spectral problems appeared in the second half of the 20th century. We mention here the works by R Beals, G Borg, LD Faddeev, MG Gasymov, IM Gelfand, BM Levitan, N Levinson, VA Marchenko, and others (see [1] for details). An important role in the inverse spectral theory for the Sturm-Liouville operator was played by the transformation operator method (see $[2,3]$ and the references therein).

At present, other effective methods for solving inverse spectral problems have been created; among them we point out the method of spectral mappings connected with ideas of the contour integration method. This method seems to offer perspective for inverse spectral problems. The created methods allowed one to solve a number of important problems in various branches of the natural sciences.

The inverse scattering theory on the half-line and on the line was studied in [4-6], and others. In [7-11] KR Mamedov studied the inverse scattering theory on the half-line with spectral parameter contained in the boundary condition. Lately, there grew interest in the 
investigation of the boundary value problem by numerical methods; e.g. [12] presented an approximate construction of the Jost function for some Sturm-Liouville boundary value problem in the case $\rho(x)=1$ by means of the collocation method; in addition, [13] is an application of spectral analysis of one-dimensional Schrödinger operators in a magnetic field. Also $[14,15]$ are applications of the discontinuous wave speed problem in a nonhomogeneous medium as in our case.

In the last 30 years there appeared many new areas for applications of inverse SturmLiouville problems, among them boundary value problems with discontinuity conditions inside the interval are connected with discontinuous material properties.

Many further applications were connected with the differential equation of the form $y^{\prime \prime}+q(x) y=r(x) y$ with turning points when the function $r(x)$ has zeros and/or changes sign. For example, we have turning points connected with physical situations in which zeros correspond to the limit of motion of a wave mechanical particle bound by a potential field. Turning points appear also in elasticity, optics, geophysics, and other branches of natural sciences. Moreover, a wide class of differential equations with Bessel-type singularities and their perturbations can be reduced to differential equations having turning points; further inverse problems for equations with turning points and singularities help one to study blow-up solutions for some nonlinear integrable evolution equations of mathematical physics (see [16]).

Inverse problems of the Sturm-Liouville equation with turning points and singularities have been studied in [17-20], and other works.

The aim of the present paper is to investigate the inverse scattering problem on the halfline $[0, \infty)$ for some version of the one-dimensional Schrödinger equation with turning. In the case of $\rho(x)=1$, the inverse problem of scattering theory for (1.1) with boundary condition not containing a spectral parameter was completely solved by Marchenko [2, 21], Levitan [3, 22], Aktosun [23], and Aktosun and Weder [24]. The discontinuous version of $\rho$ was studied by Gasymov and Levitan [25, 26], Darwish [27], and Gasymov and the author $[19,28]$. In these papers, the solution of the inverse scattering problem on the halfline $[0, \infty)$ by using the transformation operator was reduced to the solution of two inverse problems on the intervals $[0, a]$ and $[a, \infty)$. In the case $\rho \neq 1$ the inverse scattering problem was solved by the author, Guseinov, and Pashaev $[19,29]$ by using the new non-triangular representation of the Jost solution of (1.1). It turns out that in this case the discontinuity of the function $\rho(x)$ strongly influences the structure of the representation of the Jost solution and the fundamental equation of the inverse problem.

The direct and inverse problem of $(1.1)$ with $y(0)-h y(0)=0$ (see $[21,30]$ ) has been solved earlier by the so-called spectral distribution function, while the problem (1.1) with $y(0)=0$ has been studied in the works $[30,31]$ by the inverse scattering method. Furthermore, the inverse scattering problem of the one-dimensional Schröodinger's eigenvalue problem with a discontinuous coefficient was studied when $y(0)=0$ and $y^{\prime}(0)=0[27,32]$ and [25]. It should be mentioned that the spectrum of the boundary value problem (1.1)-(1.2) has been previously investigated in [33] when $\rho(x)>0$ and the boundary condition $y(0)=0$ holds.

The present paper is organized as follows. Section 1 is an introduction and preliminaries in which we demonstrate some historical and scientific survey to inverse scattering problem. We introduce, from [34], the basic definitions and results that are needed in the subsequent investigation. In addition, the scattering data for the boundary value problem 
(1.1)-(1.2) are defined and some of its spectral properties are proved. In Section 2, the main integral equation of the inverse scattering problem is derived, by its scattering data. Finally, Section 3 is devoted to a proof of the uniqueness of both the main integral equation and the solution of the inverse scattering problem.

\subsection{Preliminaries}

Consider the initial value problem

$$
\begin{aligned}
& -y^{\prime \prime}+q(x) y=\eta^{2} \rho(x) y, \quad 0 \leq x<\infty, \\
& y^{\prime}(0)-h y(0)=0, \quad h>0,
\end{aligned}
$$

where

$$
\rho(x)= \begin{cases}-1 ; & 0 \leq x \leq 1 \\ 1 ; & 1<x<\infty\end{cases}
$$

$q(x)$ is a finite real valued function which satisfies

$$
\int_{0}^{\infty}(1+x)|q(x)| d x<\infty
$$

and $\eta$ is a complex spectral parameter. In [34] the authors completely solved the direct scattering problem of the problem (1.1)-(1.4), so that, following [34] we state the main definitions and results that are needed in the subsequent investigations.

The totality of the quantities

$$
\left\{S(\eta), \eta_{k}, m_{k}, k=1,2, \ldots\right\}
$$

is called the scattering data of the problem (1.1)-(1.4). Thus, the scattering data completely determines the behavior of the normed eigenfunctions of the problem (1.1)-(1.4). Here $S(\eta)$ is the scattering function, $\eta_{k}=i \chi_{k}, \eta_{k}^{2}$ the eigenvalues and $m_{k}$ the normalizing numbers of the problem (1.1)-(1.4), where

$$
S(\eta)=\frac{f^{\prime}(0,-\eta)-h f(0,-\eta)}{f^{\prime}(0, \eta)-h f(0, \eta)},
$$

where $f^{\prime}(0, \eta)-h f(0, \eta)=0$ for $\operatorname{Re} \eta \neq 0$. From Lemmas 1.2 and 1.3 of [34], the function $S(\eta)$ is continuous on the real line $-\infty<\eta<\infty$ and admits the properties $\overline{S(\eta)}=S(-\eta)$, $|S(\eta)|=1,-\infty<\eta<\infty$. The eigenvalues $\eta_{n}^{2}=\left(i \chi_{n}\right)^{2}, \chi_{n}>0, n=1,2, \ldots$, and the normalizing numbers $m_{n}<0, n=1,2, \ldots$, satisfy the asymptotic formulas

$$
\begin{aligned}
& \eta_{n}=i \chi_{n}=i\left[\pi\left(n+\frac{1}{4}\right)-1+\frac{c_{o}}{n \pi}+o\left(\frac{1}{n}\right)\right], \quad c_{o}=\frac{1}{\pi} \int_{0}^{1} q(t) d t, \\
& m_{n}=-e^{-2 \chi_{n}}\left\{2+\frac{c_{1}}{n}+\circ\left(\frac{1}{n}\right)\right\},
\end{aligned}
$$

where

$$
c_{1}=\frac{8 h+4}{4 \pi}+\frac{3}{8 \pi} \int_{0}^{1} q(t) d t+\frac{9}{8 \pi} \int_{1}^{\infty} q(t) d t .
$$


For all $\operatorname{Re} \eta \neq 0$ we have the following equality:

$$
\frac{2 i \eta \varphi(x, \eta)}{f^{\prime}(0, \eta)-h f(0, \eta)}=f(x,-\eta)-S(\eta) f(x, \eta)
$$

where $\varphi(x, \eta)$ is the solution of the problem (1.1)-(1.2) $\left(\varphi(0, \eta)=1, \varphi^{\prime}(0, \eta)=h\right)$, and the scattering function $S(\eta)$ is given by (1.6) and satisfies the properties

$$
\overline{S(\eta)}=S(-\eta), \quad|S(\eta)|=1, \quad-\infty<\eta<\infty .
$$

In the following lemma we evaluate the asymptotic formula of the scattering function $S(\eta)$.

Lemma 1.1 For $-\infty<\eta<\infty,|\eta| \rightarrow \infty$, the scattering function $S(\eta)$ satisfies the asymptotic formula

$$
S(\eta)=S_{o}(\eta)+\bigcirc\left(\frac{1}{\eta}\right)
$$

where

$$
S_{o}(\eta)=\frac{f_{o}(0,-\eta)}{f_{o}(0, \eta)}=e^{-2 i \eta} \frac{\cosh \eta-i \sinh \eta}{\cosh \eta+i \sinh \eta} .
$$

Proof By virtue of (2.11) in [34], for $\operatorname{Im} \eta>0,|\eta| \rightarrow \infty$,

$$
\begin{aligned}
f^{\prime}(0, \eta)-h f(0, \eta) & =-i \eta f_{o}(0, \eta)+\bigcirc\left(e^{-\operatorname{Im} \eta+|\operatorname{Re} \eta|}\right), \\
\text { where } f_{o}(0, \eta) & =e^{i \eta}(\cosh \eta+i \sinh \eta),
\end{aligned}
$$

from which, in the special case for $-\infty<\eta<\infty,|\eta| \rightarrow \infty$ we have

$$
f^{\prime}(0, \eta)-h f(0, \eta)=-i \eta f_{o}(0, \eta)\left[1+\bigcirc\left(\frac{e^{|\eta|}}{\eta f_{o}(0, \eta)}\right)\right]
$$

On the other hand, it is easy to see that

$$
\left|f_{o}(0, \eta)\right| \geq \frac{e^{|\eta|}}{\sqrt{2}}
$$

from (1.15) and (1.16) we deduce, for $-\infty<\eta<\infty,|\eta| \rightarrow \infty$, that

$$
f^{\prime}(0, \eta)-h f(0, \eta)=-i \eta f_{o}(0, \eta)\left[1+\bigcirc\left(\frac{1}{\eta}\right)\right],
$$

from which, by substitution into (1.6), the proof is completed.

\section{Formulation of the inverse scattering problem}

The inverse scattering problem for the boundary value problem (1.1)-(1.4) consists of recovering the potential function $q(x)$ and the number $h$ by the scattering data. For this task to be accomplished, we must answer the following two questions:

Are the coefficient $q(x)$ and the number $h$ uniquely defined by the scattering data (1.5) of the problem (1.1)-(1.4)? 
What is the effective recovering method of $q(x)$ and $h$ by the scattering data (1.5)?

\subsection{Derivation of the main integral equation}

In this section we derive the Gelfand-Levitan integral equation of the inverse scattering problem on the interval $(1, \infty)$; this will be accomplished through Lemmas $2.1,2.2$, and Theorem 2.1. Let $\Lambda_{n}$ be the rectangular contour

$$
\Lambda_{n}=\left\{|\operatorname{Re} \eta| \leq \pi\left(n+\frac{1}{4}\right), 0 \leq \operatorname{Im} \eta \leq \pi\left(n+\frac{1}{4}\right)\right\} .
$$

Lemma 2.1 The following inequality holds true from below:

$$
|\cosh \eta+i \sinh \eta| \geq C e^{|\operatorname{Re} \eta|}, \quad \eta \in \Lambda_{n}, \forall n .
$$

Proof Let $\eta=\sigma+i \chi$, so that on the real line $\chi=0, \eta \in \Lambda_{n}$ it follows that $|\cosh \eta+i \sinh \eta|=$ $\frac{\sqrt{2}}{2} \sqrt{e^{2 \sigma}+e^{-2 \sigma}}$, from which

$$
|\cosh \eta+i \sinh \eta| \geq \frac{\sqrt{2}}{2} \sqrt{e^{2|\sigma|}}=\frac{\sqrt{2}}{2} e^{|\operatorname{Re} \eta|} .
$$

On the other hand for $\eta$ any complex number we have

$$
|\cosh \eta+i \sinh \eta| \geq \frac{\sqrt{2}}{2} \sqrt{e^{2 \sigma}+e^{-2 \sigma}-2}=\frac{\sqrt{2}}{2} e^{|\sigma|}\left(1-e^{-2|\sigma|}\right),
$$

by which, for $\sigma=\pi\left(n+\frac{1}{4}\right)$, it follows that

$$
|\cosh \eta+i \sinh \eta| \geq \frac{\sqrt{2}}{2} e^{|\operatorname{Re} \eta|}
$$

which completes the proof of the lemma.

Since, from [34], $f_{o}(0, \eta)=e^{i \eta}(\cosh \eta+i \sinh \eta)$, and using Lemma 2.1, we have

$$
\left|f_{o}(0, \eta)\right| \geq C e^{-\operatorname{Im} \eta+|\operatorname{Re} \eta|}, \quad \eta \in \Lambda_{n}, \forall n .
$$

Lemma 2.2 For every $x>1$ and $\eta \in \Lambda_{n}, n \rightarrow \infty$, we have the following asymptoticformula:

$$
\frac{-2 i \eta \varphi(x, \eta)}{f^{\prime}(0, \eta)-h f(0, \eta)}-e^{-i \eta x}-S_{o}(\eta) e^{i \eta x}=\bigcirc\left(\frac{e^{x \operatorname{Im} \eta}}{\eta}\right)
$$

where $S_{o}(\eta)$ is given by (1.12).

Proof Following [2], p.19, we see that the fundamental system $\varphi_{1}(x, \eta), \theta_{1}(x, \eta)$ of solutions of $(1.1)$, for $1<x<\infty$, with conditions $\varphi_{1}(1, \eta)=0, \varphi_{1}^{\prime}(1, \eta)=1, \theta_{1}(1, \eta)=1, \theta_{1}^{\prime}(1, \eta)=0$, have the representations

$$
\begin{aligned}
& \varphi_{1}(x, \eta)=\frac{\sin \eta(x-1)}{\eta}+\int_{1}^{x} C(x, t) \frac{\sin \eta(t-1)}{\eta} d t, \\
& \theta_{1}(x, \eta)=\cos \eta(x-1)+\int_{1}^{x} D(x, t) \cos \eta(t-1) d t,
\end{aligned}
$$


where the kernels $C(x, t)$ and $D(x, t)$ have summable first order partial derivatives [2]. For $1<x<\infty$ the solution $\varphi(x, \eta)$ of $(1.1)$ is a linear combination of $\varphi_{1}(x, \eta), \theta_{1}(x, \eta)$, so that, for $1<x<\infty$,

$$
\varphi(x, \eta)=\varphi^{\prime}(1, \eta) \varphi_{1}(x, \eta)+\varphi(1, \eta) \theta_{1}(x, \eta)
$$

We evaluate the asymptotic formulas for $\varphi_{1}(x, \eta), \theta_{1}(x, \eta)$, by integrating (2.5) and (2.6) by parts, we have

$$
\begin{aligned}
& \varphi_{1}(x, \eta)=\frac{\sin \eta(x-1)}{\eta}+\bigcirc\left(\frac{e^{(x-1) \operatorname{Im} \eta}}{\eta^{2}}\right), \\
& \theta_{1}(x, \eta)=\cos \eta(x-1)+\bigcirc\left(\frac{e^{(x-1) \operatorname{Im} \eta}}{\eta}\right) .
\end{aligned}
$$

Further, by virtue of (2.9) and (2.10) of [34], we have

$$
\begin{aligned}
& \varphi(1, \eta)=\cosh \eta+\bigcirc\left(\frac{e^{|\operatorname{Re} \eta|}}{\eta}\right), \\
& \varphi^{\prime}(1, \eta)=\eta \sinh \eta+\bigcirc\left(e^{|\operatorname{Re} \eta|}\right) .
\end{aligned}
$$

Substituting (2.8)-(2.11) into (2.7), we have

$$
\varphi(x, \eta)=\sinh \eta \sin \eta(x-1)+\cosh \eta \cos \eta(x-1)+\bigcirc\left(\frac{e^{(x-1) \operatorname{Im} \eta+|\operatorname{Re} \eta|}}{\eta}\right),
$$

when expressing $\sin \eta(x-1), \cos \eta(x-1)$, in terms of the exponential functions, (2.12) takes the form

$$
\varphi(x, \eta)=e^{i \eta x} f_{o}(0,-\eta)+e^{-i \eta x} f_{o}(0, \eta)+\bigcirc\left(\frac{e^{(x-1) \operatorname{Im} \eta+|\operatorname{Re} \eta|}}{\eta}\right),
$$

where $f_{o}(0, \eta)$ is given by (1.14). Again from (2.11) of [34], we have

$$
f^{\prime}(0, \eta)-h f(0, \eta)=-i \eta e^{i \eta}(\cosh \eta+i \sinh \eta)+\bigcirc\left(e^{(x-1) \operatorname{Im} \eta+|\operatorname{Re} \eta|}\right) .
$$

Further, from (2.14) and (2.3), we have

$$
\frac{1}{f^{\prime}(0, \eta)-h f(0, \eta)}=\frac{1}{-i \eta f_{o}(0, \eta)}\left[1+\bigcirc\left(\frac{1}{\eta}\right)\right] .
$$

From (2.13) and (2.15) we have

$$
\frac{-2 i \eta \varphi(x, \eta)}{f^{\prime}(0, \eta)-h f(0, \eta)}=\left(e^{i \eta x} S_{o}(\eta)+e^{-i \eta x}\right)\left[1+\bigcirc\left(\frac{1}{\eta}\right)\right]+\bigcirc\left(\frac{e^{(x-1) \operatorname{Im} \eta+|\operatorname{Re} \eta|}}{\eta}\right) .
$$

From (1.12), for $\eta \in \Lambda_{n}, S_{o}(\eta)$ has the asymptotic formula

$$
S_{o}(\eta)=\bigcirc\left(e^{2 \operatorname{Im} \eta}\right),
$$


by the aid of (2.17), (2.16) takes the form

$$
\frac{-2 i \eta \varphi(x, \eta)}{f^{\prime}(0, \eta)-h f(0, \eta)}=e^{-i \eta x}+S_{o}(\eta) e^{i \eta x}+\bigcirc\left(\frac{e^{x \operatorname{Im} \eta}}{\eta}\right)
$$

which completes the proof of the lemma.

The following theorem deals with the formulation of the main integral equation, the socalled (Gelfand-Levitan) integral equation of the inverse problem by its scattering data. It should be noted, here, that $\left\{S(\eta), \eta_{k}=i \chi_{k}, m_{k}\right\}$ is the scattering data of the problem (1.1)(1.2) and $\left\{S_{o}(\eta), \eta_{k}^{o}=i \chi_{k}^{o}, m_{k}^{o}\right\}$ is the scattering data of the problem (1.1)-(1.2) with $q(x)=0$, $h=0, S_{o}(\eta)$ is given by $(1.12)$

$$
m_{k}^{o}=-e^{-2 \chi_{k}^{o},} \quad \chi_{k}^{o}=\pi\left(k+\frac{1}{4}\right)
$$

Theorem 2.1 For $x>1$, we have the following integral equation:

$$
H(x+y)+K(x, y)+\int_{x}^{\infty} K(x, y) H(x, y) d y=0 \quad(x \leq y<\infty)
$$

where

$$
\begin{aligned}
H(x)= & \sum_{k=1}^{\infty}\left(h \frac{e^{-\chi_{k} x}}{m_{k}}-\frac{e^{-\chi_{k}^{o} x}}{m_{k}^{o}}\right) \\
& +\int_{-\infty}^{\infty}\left\{S_{o}(\eta)-S(\eta)\right\} e^{i \eta x} d \eta, \quad x>2,
\end{aligned}
$$

$S_{o}(\eta), \chi_{k}^{o}$ and $m_{k}^{o}$ are given by (2.18).

Proof Following (1.32) and (1.14) of [34], we have

$$
\begin{aligned}
& \frac{2 i \eta \varphi(x, \eta)}{f^{\prime}(0, \eta)-h f(0, \eta)}=f(x,-\eta)-S(\eta) f(x, \eta), \\
& f(x, \lambda)=e^{i \lambda x}+\int_{x}^{\infty} K(x, t) e^{i \lambda t} d t, \quad 1<x<\infty
\end{aligned}
$$

where $\operatorname{Im} \lambda \geq 0, K(x, x)=\frac{1}{2} \int_{0}^{x} q(t) d t, 1<x<\infty$.

Substituting (2.22) into (2.21), we have

$$
\begin{aligned}
& \frac{2 i \eta \varphi(x, \eta)}{f^{\prime}(0, \eta)-h f(0, \eta)}-e^{-i \eta x}+S_{o}(\eta) e^{i \eta x} \\
& =\left\{S_{o}(\eta)-S(\eta)\right\} e^{i \eta x}+\int_{x}^{\infty} K(x, t) e^{-i \eta t} d t \\
& \quad+S_{o}(\eta) \int_{x}^{\infty} K(x, t) e^{i \eta t} d t \\
& \quad+\left\{S_{o}(\eta)-S(\eta)\right\} \int_{x}^{\infty} K(x, t) e^{i \eta t} d t .
\end{aligned}
$$


Multiplying both sides of (2.23) by $\frac{e^{i n y}}{2 \pi}$ and integrating with respect to $\eta$ on $[-N, N]$ where $N$ is a positive integer, we have

$$
\begin{aligned}
\frac{1}{2 \pi} \int_{-N}^{N}\left[\frac{2 i \eta \varphi(x, \eta)}{f^{\prime}(0, \eta)-h f(0, \eta)}-e^{-i \eta x}+S_{o}(\eta) e^{i \eta x}\right] e^{i \eta y} d \eta \\
=\frac{1}{2 \pi} \int_{-N}^{N}\left[\left\{S_{o}(\eta)-S(\eta)\right\} e^{i \eta(x+y)}\right] d \eta \\
\quad+\frac{1}{2 \pi} \int_{-N}^{N}\left\{\int_{x}^{\infty} K(x, t) e^{-i \eta t} d t\right\} e^{i \eta y} d \eta \\
\quad-\frac{1}{2 \pi} \int_{-N}^{N}\left[S_{o}(\eta)\left\{\int_{x}^{\infty} K(x, t) e^{i \eta t} d t\right\} e^{i \eta y}\right] d \eta \\
\quad+\frac{1}{2 \pi} \int_{-N}^{N}\left[\left\{S_{o}(\eta)-S(\eta)\right\}\left\{\int_{x}^{\infty} K(x, t) e^{-i \eta t} d t\right\}\right] e^{i \eta \eta y} d \eta .
\end{aligned}
$$

For convenience, we write (2.24) in the following abbreviated form:

$$
I_{\mathrm{C} 11}=I_{\mathrm{FP} 1}+I_{\mathrm{FP} 2}-I_{\mathrm{C} 12}+I_{\mathrm{FP} 3}
$$

where

$$
\begin{aligned}
& I_{\mathrm{CI} 1}=\frac{1}{2 \pi} \int_{-N}^{N}\left[\frac{2 i \eta \varphi(x, \eta)}{f^{\prime}(0, \eta)-h f(0, \eta)}-e^{-i \eta x}+S_{o}(\eta) e^{i \eta x}\right] e^{i \eta y} d \eta, \\
& I_{\mathrm{FP} 1}=\frac{1}{2 \pi} \int_{-N}^{N}\left[\left\{S_{o}(\eta)-S(\eta)\right\} e^{i \eta(x+y)}\right] d \eta, \\
& I_{\mathrm{FP} 2}=\frac{1}{2 \pi} \int_{-N}^{N}\left\{\int_{x}^{\infty} K(x, t) e^{-i \eta t} d t\right\} e^{i \eta y} d \eta, \\
& I_{\mathrm{CI} 2}=\frac{1}{2 \pi} \int_{-N}^{N}\left[S_{o}(\eta)\left\{\int_{x}^{\infty} K(x, t) e^{i \eta t} d t\right\} e^{i \eta y}\right] d \eta, \\
& I_{\mathrm{FP} 3}=\frac{1}{2 \pi} \int_{-N}^{N}\left[\left\{S_{o}(\eta)-S(\eta)\right\}\left\{\int_{x}^{\infty} K(x, t) e^{-i \eta t} d t\right\}\right] e^{i \eta \eta y} d \eta .
\end{aligned}
$$

We calculate $I_{\mathrm{Cl} 1}, I_{\mathrm{Cl} 2}$ by means of contour integration over the contour $\Lambda_{n}$, whereas, for $I_{\mathrm{FP} 1}, I_{\mathrm{FP} 2}$, and $I_{\mathrm{FP} 3}$, we use the Fourier-Plancherel formulas. First for $I_{\mathrm{FP} 1}$, we see from (1.11) that the function $\left[S_{o}(\eta)-S(\eta)\right] \in L_{2}(-\infty, \infty)$, consequently, by the Fourier-Plancherel theorem, there exists a limiting function $H_{S}(x) \in L_{2}(-\infty, \infty)$, where

$$
H_{S}(x) \stackrel{\text { def }}{=} \frac{1}{2 \pi} \int_{-\infty}^{\infty}\left[\left\{S_{o}(\eta)-S(\eta)\right\} e^{i \eta x}\right] d \eta
$$

and, consequently,

$$
\lim _{N \rightarrow \infty} I_{\mathrm{FP} 1}=H_{S}(x+y) \stackrel{\text { def }}{=} \frac{1}{2 \pi} \int_{-\infty}^{\infty}\left[\left\{S_{o}(\eta)-S(\eta)\right\} e^{i \eta(x+y)}\right] d \eta .
$$

Further, with the aid of the inversion formula of Fourier-Plancherel, we see that

$$
\lim _{N \rightarrow \infty} \frac{1}{2 \pi} \int_{-N}^{N}\left\{\int_{x}^{\infty} K(x, t) e^{-i \eta t} d t\right\} e^{i \eta y} d \eta=K(x, y)
$$


exists and hence

$$
\begin{aligned}
& \lim _{N \rightarrow \infty} I_{\mathrm{FP} 2}=K(x, y), \\
& \lim _{N \rightarrow \infty} I_{\mathrm{FP} 3}=\int_{x}^{\infty} K(x, t) H_{S}(t+y) d t .
\end{aligned}
$$

Now, by using the contour integration method, we calculate $I_{\mathrm{CI} 1}, I_{\mathrm{CI} 2}$.

Let $N_{n}=\pi\left(n+\frac{1}{4}\right)$, where $n$ is an arbitrary positive integer, and $\Lambda_{n}^{+}=\Lambda_{n}-[-\pi(n+$ $\left.\frac{1}{4}\right), \pi\left(n+\frac{1}{4}\right)$ ], i.e. $\Lambda_{n}^{+}$is the part of the contour $\Lambda_{n}$ which lies in the upper half of imaginary axis, $\operatorname{Im} \eta>0$. For simplicity, denote

$$
\Theta(x, y, \eta)=S_{o}(\eta)\left\{\int_{x}^{\infty} K(x, t) e^{-i \eta t} d t\right\} e^{i \eta y} .
$$

The function $\Theta(x, y, \eta)$ is analytic function in the half plane $\operatorname{Im} \eta>0$ except for its poles $\eta_{k}^{o}=i \chi_{k}^{o}$. We have

$$
\frac{1}{2 \pi i} \oint_{\Lambda_{n}} \Theta(x, y, \eta) d \eta=\frac{1}{2 \pi i} \int_{-N_{n}}^{N_{n}} \Theta(x, y, \eta) d \eta+\frac{1}{2 \pi i} \int_{\Lambda_{n}^{+}} \Theta(x, y, \eta) d \eta
$$

We prove that

$$
\lim _{n \rightarrow \infty} \int_{\Lambda_{n}^{+}} \Theta(x, y, \eta) d \eta=0
$$

in fact, using integration by parts we see that

$$
\int_{x}^{\infty} K(x, t) e^{-i \eta t} d t=\bigcirc\left(\frac{e^{-x \operatorname{Im} \eta}}{\eta}\right)
$$

Further, from (2.17), (2.35), and (2.38), we have

$$
\Theta(x, y, \eta)=\bigcirc\left(\frac{e^{-(x+y-2) \operatorname{Im} \eta}}{\eta}\right), \quad \eta \in \Lambda_{n}^{+},
$$

on the vertical part of $\Lambda_{n}^{+}$, for $\eta \in \Lambda_{n}^{+}$, we have

$$
\begin{aligned}
& \left|\int_{0}^{\pi\left(n+\frac{1}{4}\right)} \bigcirc\left(\frac{e^{-(x+y-2) \operatorname{Im} \eta}}{\eta}\right) d \eta\right| \\
& \quad \leq \frac{\operatorname{costant}}{\pi\left(n+\frac{1}{4}\right)} \frac{1-e^{-(x+y-2) \pi\left(n+\frac{1}{4}\right)}}{(x+y-2)} \rightarrow 0, \quad n \rightarrow \infty .
\end{aligned}
$$

Similarly, for $\eta \in$ horizontal part of $\Lambda_{n}^{+}$, we have

$$
\left|\int_{-\pi\left(n+\frac{1}{4}\right)}^{\pi\left(n+\frac{1}{4}\right)} \bigcirc\left(\frac{e^{-(x+y-2) \pi\left(n+\frac{1}{4}\right)}}{|\eta|}\right) d \eta\right| \rightarrow 0, \quad n \rightarrow \infty,
$$

the proof of (2.37) is completed from (2.40) and (2.41). 
With the aid of the well-known residue formula, we have

$$
\begin{aligned}
& \frac{1}{2 \pi i} \oint_{\Lambda_{n}} \Theta(x, y, \eta) d \eta \\
& \quad=\sum_{k=1}^{n} \operatorname{Res}_{\eta=\eta_{k}^{o}} \Theta(x, y, \eta)=-\sum_{k=1}^{n} \frac{f_{o}\left(0,-\eta_{k}^{o}\right)}{\dot{f}_{o}\left(0, \eta_{k}^{o}\right)} \int_{x}^{\infty} K(x, t) e^{i \eta_{k}^{o}(t+y)} d t,
\end{aligned}
$$

from (1.14) it follows that $\frac{f_{o}\left(0,-\eta_{k}^{o}\right)}{\dot{f}_{o}\left(0, \eta_{k}^{o}\right)}=i e^{2 \chi_{k}^{o}}$, for $\eta_{k}^{o}=i \chi_{k}^{o}$, from this (2.42) becomes

$$
\begin{aligned}
& \lim _{n \rightarrow \infty} \frac{1}{2 \pi i} \oint_{\Lambda_{n}} \Theta(x, y, \eta) d \eta \\
& \quad=-i \int_{x}^{\infty}\left\{\sum_{k=1}^{\infty} \frac{e^{-i \chi_{k}^{o}(t+y)}}{m_{k}^{o}} K(x, t)\right\} d t, \quad \text { where } m_{k}^{o}=-e^{-2 x_{k}^{o}}
\end{aligned}
$$

From (2.37) and (2.43), (2.36) takes the form

$$
\lim _{n \rightarrow \infty} I_{\mathrm{CI} 2}=-i \int_{x}^{\infty}\left\{\sum_{k=1}^{\infty} \frac{e^{-i \chi_{k}^{o}(t+y)}}{m_{k}^{o}} K(x, t)\right\} d t .
$$

We evaluate $I_{\mathrm{CI} 1}$. Let

$$
\Phi(x, y, \eta)=\left[\frac{2 i \eta \varphi(x, \eta)}{f^{\prime}(0, \eta)-h f(0, \eta)}-e^{-i \eta x}+S_{o}(\eta) e^{i \eta x}\right] e^{i \eta y}
$$

consider the integration

$$
\frac{1}{2 \pi i} \oint_{\Lambda_{n}} \Phi(x, y, \eta) d \eta=\frac{1}{2 \pi i} \int_{-N_{n}}^{N_{n}} \Phi(x, y, \eta) d \eta+\frac{1}{2 \pi i} \int_{\Lambda_{n}^{+}} \Phi(x, y, \eta) d \eta
$$

In a similar way to the integration $I_{\mathrm{C} 12}$, it can be seen that $\lim _{n \rightarrow \infty} \frac{1}{2 \pi i} \int_{\Lambda_{n}^{+}} \Phi(x, y, \eta) d \eta=0$. Using the residue formula we have

$$
\begin{aligned}
\frac{1}{2 \pi i} \oint_{\Lambda_{n}} \Phi(x, y, \eta) d \eta= & \frac{1}{2 \pi i} \oint_{\Lambda_{n}}\left[\frac{2 i \eta \varphi(x, \eta)}{f^{\prime}(0, \eta)-h f(0, \eta)}-e^{-i \eta x}\right] e^{i \eta y} d \eta \\
& +\frac{1}{2 \pi i} \oint_{\Lambda_{n}} S_{o}(\eta) e^{i \eta(x+y)} d \eta .
\end{aligned}
$$

It is easy to see that

$$
\begin{aligned}
& \operatorname{Res}_{\eta=\eta_{k}^{o}}\left(S_{o}(\eta) e^{i \eta(x+y)}\right)=\frac{-i}{m_{k}^{o}} e^{i \chi_{k}^{o}(x+) y}, \\
& \operatorname{Res}_{\eta=\eta_{k}^{o}}\left[\frac{2 i \eta \varphi(x, \eta)}{f^{\prime}(0, \eta)-h f(0, \eta)}-e^{-i \eta x}\right] e^{i \eta y} \\
& \quad=\frac{i f\left(x, \eta_{k}\right)}{m_{k}} e^{i \eta_{k} y}=\frac{h}{m_{k}} e^{-\chi_{k}(x+y)}+h \int_{x}^{\infty} K(x, t) \frac{1}{m_{k}} e^{-\chi_{k}(x+y)} d t .
\end{aligned}
$$


Substituting (2.48) into (2.47), we get

$$
\begin{aligned}
& \frac{1}{2 \pi i} \oint_{\Lambda_{n}} \Phi(x, y, \eta) d \eta \\
& \quad=i \sum_{k=1}^{n}\left\{\frac{-e^{i \chi_{k}^{o}(x+) y}}{m_{k}^{o}}+\frac{h e^{-\chi_{k}(x+y)}}{m_{k}}+h \int_{x}^{\infty} K(x, t) \frac{e^{-\chi_{k}(x+y)}}{m_{k}} d t\right\} .
\end{aligned}
$$

From (2.49), as $n \rightarrow \infty$, (2.29) becomes

$$
\lim _{n \rightarrow \infty} I_{\mathrm{CI} 1}=i \sum_{k=1}^{\infty}\left\{\frac{-e^{i \chi_{k}^{o}(x+) y}}{m_{k}^{o}}+\frac{h e^{-\chi_{k}(x+y)}}{m_{k}}+h \int_{x}^{\infty} K(x, t) \frac{e^{-\chi_{k}(x+y)}}{m_{k}} d t\right\} .
$$

By putting $N=N_{n}$ in (2.25), passing to the limit as $n \rightarrow \infty$, and taking into account (2.32), (2.33), (2.34), (2.44), and (2.50), we have

$$
H(x+y)+K(x, y)+\int_{x}^{\infty} K(x, t) H(t+y) d t=0 \quad(1<x<y<\infty)
$$

The integral equation (2.51) is still valid for $x=y$, this can be proved by continuity of $K(x, y) \leq C \sigma\left(\frac{x+y}{2}\right)$ and the continuity of $H(\xi)$ in the metric $L_{2}(2, \infty)$.

\section{The uniqueness theorems}

In this section we prove two theorems, first, the uniqueness theorem of the solution of the main integral equation $(2.51)$ on the interval $(1, \infty)$, the second is the uniqueness of the inverse scattering problem of (1.1)-(1.2) by its scattering data (1.5).

\subsection{The uniqueness theorem of the main integral equation}

We prove the uniqueness of the solution of the integral equation (2.51) with respect to $K(x, t)$.

Theorem 3.1 For every fixed $x>1$, the solution $\beta(y) \in L^{2}(x, \infty)$ of the equation

$$
\alpha(y)+\beta(y)+\int_{x}^{\infty} \beta(t) H(t+y) d t=0 \quad(x \leq y<\infty)
$$

is unique, where $\alpha(y) \in L^{2}(x, \infty)$ is a given function

Proof It is sufficient to prove that the homogeneous integral equation

$$
\beta(y)+\int_{x}^{\infty} \beta(t) H(t+y) d t=0 \quad(x \leq y<\infty)
$$

has only the zero solution $\beta(y) \equiv 0$ in the space $L^{2}(x, \infty)$.

Beside the boundary value problem (1.1)-(1.2), we consider the following boundary value problem:

$$
\begin{array}{ll}
-y^{\prime \prime}+q(x) y=z y, & 0 \leq x<\infty \\
y^{\prime}(0)-h y(0)=0, & h>0,
\end{array}
$$


with the same function $q(x)$ of (1.1). We denote by $J(x, \eta)$, where $z=\eta^{2}$, the Jost solution of (3.3), with the property

$$
J(x, \eta)=e^{i \eta x}+o(1), \quad \text { for } x \rightarrow \infty(-\infty<\eta<\infty) .
$$

We see, from [2], that this solution, uniquely, exists and has the representation

$$
J(x, \eta)=e^{i \eta x}+\int_{x}^{\infty} \widetilde{K}(x, t) e^{i \eta t} d t .
$$

It is clear (1.1) and (3.3) are the same for $1<x<\infty$ and satisfy the same condition (3.4). We deduce, from the last discussion, that $f(x, \eta)=J(x, \eta), 1<x<\infty$, from which, using (2.22), (3.6), we obtain

$$
K(x, t)=\widetilde{K}(x, t), \quad \text { for } 1<x \leq t<\infty .
$$

Let $\widetilde{S}(\eta)$ denotes the scattering function of (3.3)-(3.4). From [2], and keeping in mined that the problem (3.3)-(3.4) has no negative eigenvalues, it can be seen that

$$
\widetilde{H}(x+y)+\widetilde{K}(x, y)+\int_{x}^{\infty} \widetilde{K}(x, t) \widetilde{H}(t+y) d t=0 \quad(x \leq y<\infty),
$$

where $\widetilde{H}(x)=\frac{1}{2 \pi} \int_{-\infty}^{\infty}\{1-\widetilde{S}(\eta)\} e^{i \eta x} d \eta$. Subtracting (3.8) from (2.51) and using (3.7), we obtain

$$
H(x+y)-\widetilde{H}(x+y)+\int_{x}^{\infty} K(x, t)\{H(t+y)-\widetilde{H}(t+y)\} d t=0 \quad(1<x \leq y<\infty) .
$$

Putting $y=x$ in (3.9), we have the following Volterra homogeneous integral equation:

$$
H(2 x)-\widetilde{H}(2 x)+\int_{x}^{\infty} K(x, 2 \zeta-x)\{H(2 \zeta)-\widetilde{H}(2 \zeta)\} d \zeta=0 \quad(1<x<\infty),
$$

which has the zero solution with respect to $H(2 x)-\widetilde{H}(2 x)$, so that

$$
H(2 \zeta) \equiv \widetilde{H}(2 \zeta), \quad \text { for } 2<\zeta<\infty,
$$

from which (3.2) is written in the form

$$
\beta(y)+\int_{x}^{\infty} \beta(t) \tilde{H}(t+y) d t=0 \quad(x \leq y<\infty) .
$$

By virtue of [2], the last equation has only the zero solution $\beta(y)=0$.

\subsection{The uniqueness theorem of inverse scattering problem}

Theorem 3.2 The scattering data

$$
\left\{S(\eta)(-\infty<\eta<\infty), \eta_{n}=i \chi_{n}, m_{n}(n=1,2, \ldots)\right\}
$$

of the problem (1.1)-(1.2) uniquely defines the potential $q(x)$ of (1.1) and the number $h$ of the condition (1.2). 
Proof In Theorem 2.1, we have constructed the function $H(x), x>2$, and used it to derive the main integral equation by the scattering data (3.13). From (2.22) we have $q(x)=$ $-2 \frac{d}{d x} K(x, t)$, also from Theorem 3.1, the function $K(x, t)$ is unique for $1<x<\infty$ and consequently the potential $q(x), 1<x<\infty$, is unique. As a consequence of the uniqueness of $q(x)$ the functions $f(1, \eta)$ and $f^{\prime}(1, \eta)$ are uniquely defined.

We prove, now, the uniqueness of $q(x)$ for $0 \leq x \leq 1$. Consider the solution $\phi(x, \eta)$, $0 \leq x \leq 1$, of the problem (1.1) satisfying the initial conditions $\phi(0, \eta)=1, \phi^{\prime}(0, \eta)=h$. Let $W[f, \phi]$ be the Wronskian of the two independent solutions $f(x, \eta), \phi(x, \eta)$. By virtue of $\left.W\right|_{x=1}=\left.W\right|_{x=0}$, we have

$$
f(1, \eta) \phi(1, \eta)-f^{\prime}(1, \eta) \phi(1, \eta)=-\left[f^{\prime}(0, \eta)-h f(0, \eta)\right] .
$$

Replacing $\eta$ by $-\eta$ and keeping in mind that $\phi(x, \eta)=\phi(x,-\eta)$, we have

$$
f(1,-\eta) \phi(1, \eta)-f^{\prime}(1,-\eta) \phi(1, \eta)=-\left[f^{\prime}(0,-\eta)-h f(0,-\eta)\right] .
$$

Solving (3.14) and (3.15) for $\phi(1, \eta), \phi^{\prime}(1, \eta)$, then dividing the results by $f^{\prime}(0, \eta)-h f(0, \eta)$, we obtain

$$
\begin{aligned}
& \frac{\phi(1, \eta)}{f^{\prime}(0, \eta)-h f(0, \eta)}=\frac{-1}{2 i \eta}\{f(1,-\eta)-S(\eta) f(1, \eta)\}, \\
& \frac{\phi^{\prime}(1, \eta)}{f^{\prime}(0, \eta)-h f(0, \eta)}=\frac{-1}{2 i \eta}\left\{f^{\prime}(1,-\eta)-S(\eta) f^{\prime}(1, \eta)\right\},
\end{aligned}
$$

from which we have

$$
N(\eta) \stackrel{\text { def }}{=} \frac{\phi(1, \eta)}{\phi^{\prime}(1, \eta)}=\frac{f(1,-\eta)-S(\eta) f(1, \eta)}{f^{\prime}(1,-\eta)-S(\eta) f^{\prime}(1, \eta)} .
$$

It should be noted, here, that the function $N(\eta)$ defined by (3.17) is well defined because of the uniqueness of $f(1, \eta), f^{\prime}(1, \eta)$. The functions $\phi(1, \eta), \phi^{\prime}(1, \eta)$ are entire functions, and then $N(\eta)$ is a meromorphic function for $-\infty<\eta<\infty$; indeed, by analytic continuation, it is meromorphic in the whole complex plane. Further, the zeros and poles of $N(\eta)$ are the roots of the equations

$$
\begin{aligned}
& \phi(1, \eta)=0, \\
& \phi^{\prime}(1, \eta)=0,
\end{aligned}
$$

respectively. On the other hand, (3.18), (3.19) are regarded as the characteristic equations of the two boundary value problems

$$
\begin{aligned}
& -y^{\prime \prime}+q(x) y=-\eta^{2} y, \quad 0 \leq x \leq 1, \\
& y(0)=0, \quad y(1)=0, \\
& -y^{\prime \prime}+q(x) y=-\eta^{2} y, \quad 0 \leq x \leq 1, \\
& y(0)=0, \quad y^{\prime}(1)=0 .
\end{aligned}
$$


The two spectra of (3.20) and (3.21) can be considered known, so that, arguing as in [26], we deduce the uniqueness of $q(x), 0 \leq x \leq 1$. It remains to prove that the number $h$ of the boundary condition (1.2) is uniquely defined by (3.13).

Beside the boundary value problem (1.1)-(1.2), consider the boundary value problem of (1.1) subject to the initial condition

$$
y^{\prime}(0)-h_{1} y(0)=0
$$

with the same weight function $\rho(x)$ and potential $q(x)$ of (1.1). Denote by $\left\{\eta_{n}\right\}_{n=1}^{\infty}$ and $\left\{\widetilde{\eta}_{n}\right\}_{n=1}^{\infty}$, the eigenvalues of (1.1)-(1.2) and (1.1)-(3.22), respectively. Let the characteristic equations of (1.1)-(1.2) and (1.1)-(3.22) be $\Phi(\eta)=0$ and $\Phi_{1}(\eta)=0$, respectively, where

$$
\begin{aligned}
& \Phi(\eta)=f^{\prime}(0, \eta)-h f(0, \eta), \\
& \Phi_{1}(\eta)=f^{\prime}(0, \eta)-h_{1} f(0, \eta),
\end{aligned}
$$

where $f(x, \eta)$ is the solution of (1.1)-(1.2) subject to $f(0, \eta)=1, f^{\prime}(0, \eta)=h$. From (2.21) of [34] and (3.24) we have

$$
m_{n}=\frac{\dot{\Phi}(\eta) f\left(0, \eta_{n}\right)}{2 \eta_{n}}
$$

From (3.23), (3.24), and (3.25) we have

$$
h-h_{1}=-\frac{\dot{\Phi}(\eta) \Phi_{1}(\eta)}{2 \eta_{n} m_{n}} .
$$

The functions $\dot{\Phi}(\eta)$ and $\Phi_{1}(\eta)$ as entire functions are written in the form

$$
\begin{aligned}
& \Phi(\eta)=C \prod_{n=0}^{\infty}\left(1-\frac{\eta}{\eta_{n}}\right), \\
& \Phi_{1}(\eta)=C_{1} \prod_{n=0}^{\infty}\left(1-\frac{\eta}{\widetilde{\eta}_{n}}\right),
\end{aligned}
$$

where the constants $C, C_{1}$ are uniquely defined with the help of the asymptotic formulas

$$
\lim _{\operatorname{Im} \eta \rightarrow \infty} \frac{\Phi(\eta)}{\eta W_{o}(\eta)}=\lim _{\operatorname{Im} \eta \rightarrow \infty} \frac{\Phi_{1}(\eta)}{\eta W_{o}(\eta)}=-i
$$

and $W_{o}(\eta)=e^{i \eta}(\cosh \eta+i \sinh \eta)$. From (3.26) and (3.27) we see that $h-h_{1}$ is uniquely defined; moreover, $h$ is uniquely defined by (1.19) of [34], $h=A(0,0)$, so that $h_{1}$ is uniquely defined, which completes the proof.

The authors declare that they have no competing interests. 


\section{Acknowledgements}

We are indebted to an anonymous referee for a detailed reading of the manuscript and useful comments and suggestions, which helped us improve this work. This work is supported by Office of Scientific Publishing Excellence of Alexandria University.

\section{Received: 3 December 2014 Accepted: 12 March 2015 Published online: 03 April 2015}

\section{References}

1. Freiling, G, Yurko, VA: Inverse Sturm-Liouville Problems and Their Applications. Nova Science Publishers, New York (2001)

2. Marchenko, VA: Sturm-Liouville Operators and Applications. Operator Theory: Advances and Applications, vol. 22. Birkhäuser, Basel (1986)

3. Levitan, BM: On the solution of the inverse problem of quantum scattering theory. Math. Notes 17(4), 611-624 (1975)

4. Faddeev, LD: On a connection of the S-matrix with the potential for the one-dimensional Schrödinger operator. Dokl. Akad. Nauk SSSR 121, 63-66 (1958)

5. Agranovich, ZS, Marchenko, VA: The Inverse Problem of Scattering Theory. Gordon \& Breach, New York (1963)

6. Kurasov, P: Scattering matrices with finite phase shift and the inverse scattering problem. Inverse Probl. 12(3), 295-307 (1996)

7. Mamedov, KR: On an inverse scattering problem for a discontinuous Sturm-Liouville equation with a spectral parameter in the boundary condition. Bound. Value Probl. 2010, Article ID 171967 (2010)

8. Mamedov, KR: Uniqueness of the solution to the inverse problem of scattering theory for the Sturm-Liouville operator with a spectral parameter in the boundary condition. Math. Notes 74(1), 136-140 (2003)

9. Mamedov, KR: Uniqueness of solution of the inverse problem of scattering theory for Sturm-Liouville operator with discontinuous coefficient. Proc. Inst. Math. Mech. Natl. Acad. Sci. Azerb. 24(32), 163-172 (2006)

10. Mamedov, KR, Kosar, NP: Inverse scattering problem for Sturm-Liouville operator with nonlinear dependence on the spectral parameter in the boundary condition. Math. Methods Appl. Sci. 34(2), 231-241 (2010)

11. Mamedov, KR, Menken, $\mathrm{H}$ : The Levinson-type formula for a boundary value problem with a spectral parameter in the boundary condition. Arab. J. Sci. Eng. 34(1), 219-226 (2009)

12. Aliev, AR, Gasymova, SG, Gasymova, DG, Ahmadzadeh, ND: Approximate construction of the Jost function by the collocation method for Sturm-Liouville boundary value problem. Azerb. J. Math. 3(2), 45-61 (2013)

13. Aliev, AR, Eyvazov, EH: The resolvent equation of the one-dimensional Schrödinger operator on the whole axis. Sib. Mat. Zh. 53(6), 1201-1208 (2012); English transl.: Sib. Math. J. 53(6), 957-964 (2012)

14. Efendiev, RF, Orudzhev, HD: Inverse wave spectral problem with discontinuous wave speed. Zh. Mat. Fiz. Anal. Geom. 6(3), 255-265 (2010)

15. Efendiev, RF: Spectral analysis for one class of second-order indefinite non-self-adjoint differential operator pencil. Appl. Anal. 90(12), 1837-1849 (2011)

16. Constantin, A: On the inverse spectral problem for the Camassa-Holm equation. J. Funct. Anal. 155(2), 352-363 (1998)

17. Boumenir, A: Inverse spectral problem for the Laguerre differential operator. J. Math. Anal. Appl. 224(2), 218-240 (1998)

18. Carlson, R: Inverse spectral theory for some singular Sturm-Liouville problems. J. Differ. Equ. 106, 121-140 (1993)

19. El-Reheem, ZFA: On the scattering problem for the Sturm-Liouville equation on the half-line with sign valued weight coefficient. Appl. Anal. 57, 333-339 (1995)

20. Yurko, VA: On recovering Sturm-Liouville differential operators with singularities inside the interval. Mat. Zametki 64(1), 143-156 (1998): English transl.: Math. Notes 64(1), 121-132 (1998)

21. Marchenko, VA: On reconstruction of the potential energy from phases of the scattered waves. Dokl. Akad. Nauk SSSR 104, 695-698 (1955)

22. Levitan, BM: Inverse Sturm-Liouville Problems. VSP, Zeist (1987)

23. Aktosun, T: Construction of the half-line potential from the Jost function. Inverse Probl. 20(3), 859-876 (2004)

24. Aktosun, T, Weder, R: Inverse spectral-scattering problem with two sets of discrete spectra for the radial Schrödinger equation. Inverse Probl. 22(1), 89-114 (2006)

25. Gasymov, MG: Direct and inverse problem of spectral analysis for a class of equations with a discontinuous coefficient. In: Lavrent'ev, MM (ed.) Non-Classical Methods in Geophysics, pp. 37-44. Nauka, Novosibirsk (1977)

26. Gasymov, MG, Levitan, BM: Determination of o differential equation by two of its spectra. Usp. Mat. Nauk 19(2), 3-63 (1964); English transl.: Russ. Math. Surv. 19, 1-64 (1964)

27. Darwish, AA: The inverse scattering problem for a singular boundary value problem. N.Z. J. Math. 23, 37-56 (1994)

28. Gasymov, MG, El-Reheem, ZFA: On the theory of inverse Sturm-Liouville problems with discontinuous sign-alternating weight. Dokl. Akad. Nauk Azerb. 48-50(1-12), 13-16 (1996)

29. Guseinov, IM, Pashaev, RT: On an inverse problem for a second-order differential equation. Usp. Mat. Nauk 57(3(345)), 147-148 (2002)

30. Naimark, MA: Linear Differential Operators. Ungar, New York (1967)

31. Binding, PA, Browne, PJ, Watson, BA: Spectral isomorphisms between generalized Sturm-Liouville problems. In: Linear Operators and Matrices. Operator Theory: Advances and Applications, vol. 130, pp. 135-152 (2002)

32. Pashaev, ET: Uniqueness theorem inverse problem and spectral theory for one class system differential equation with discontinuous coefficient. Dokl. Akad. Nauk Azerb. SSR 35, 57-73 (1979)

33. Eugene, B: Mathematical Physics. Addison-Wesley, Reading (1973)

34. EI-Reheem, ZFA, Nasser, AH: On the spectral investigation of the scattering problem for some version of one-dimensional Schrödinger equation with turning point. Bound. Value Probl. 2014, 97 (2014) 